Vietnam Journal of Mechanics, VAST, Vol. 27, No. 4 (2005), pp. 213-219

\title{
APPLICATION OF GENERALIZED DIFFUSION THEORY FOR CALCULATION OF PARTICLE DISTRIBUTION IN SUSPENSION FLOW BETWEEN TWO VERTICALLY COAXIAL CYLINDERS
}

\author{
NGUYEN HONG PHAN \\ Institute of Mechanics
}

\begin{abstract}
Applications of the generalized diffusion theory for calculation of rotating spherical particle distribution in a suspension with viscouse fluid, using nonlinear closing relationships are demonstrated. A problem of motion of suspension in the gap between two rotating vertically coaxial cylinders was established and stationary case of the problem was numerically investigated. A comparison between the numerical prediction of particle concentration and the experimental data of Phillips et al. (1992) was made.
\end{abstract}

\section{GOVERNING EQUATIONS}

The flow of viscous fluid carrying rigid spherical rotating particles, that have densitity coincided with density of the fluid, is decribed by the following equation system [1]:

$$
\begin{aligned}
& \bar{\nabla} \cdot \bar{U}=0 ; \quad \bar{U}=\varphi \bar{U}_{1}+(1-\varphi) \bar{U}_{2} ; \\
& \rho \frac{d \varphi}{d t}=-\bar{\nabla} \cdot \bar{J} ; \quad \bar{J}=\rho \varphi\left(\bar{U}_{1}-\bar{U}\right) ; \quad \rho=\mathrm{const} ; \\
& \rho \frac{d \bar{U}}{d t}=\rho \bar{g}-\bar{\nabla} p-\bar{\nabla} \times \bar{\tau}^{A}+\bar{\nabla} \cdot \bar{\tau}^{S} ; \\
& j \frac{d \bar{\omega}}{d t}+j\left(\frac{\bar{J}}{A} \cdot \bar{\nabla}\right) \bar{\omega}=-\bar{\tau}^{A}+\bar{\nabla} \times \lambda^{A}+\bar{\nabla} \cdot \bar{\lambda}^{S} ; \quad j=n \frac{4}{5} \pi R^{5} \rho ; \quad A=\rho \varphi ; \\
& \frac{D \bar{J}}{D t}=-\rho \varphi(1-\varphi)\left[\bar{F}-\frac{1}{1-\varphi}\left(\bar{\nabla} \mu_{1}\right)_{p, T}\right],
\end{aligned}
$$

where $\bar{U}_{1}, \bar{U}_{2}, \bar{U}$ - mean volume velocities of particles (dispersed phase), fluid (liquid phase) and suspension (mixture), respectively; $\rho$ - common density of particles and fluid; $\varphi$ - volume concentration of particles; $\bar{J}$ - generalized diffusion flux of particles; $\bar{\omega}$ - mean angular velocity of particles; $j$ - particle moment of inertia; $n$ - number of particle in an unit volume of suspension; $\mu_{1}$ - generalized chemical potential of particles; $\bar{F}$-generalized diffusion force; $p$ - thermodynamical pressure; $\bar{\tau}^{A}, \bar{\tau}^{S}$ - antisymmetric and symmetric parts of viscous stress tensor; $\bar{\lambda}^{A}, \bar{\lambda}^{S}$ - antisymmetric and symmetric parts of moment stress tensor.

The nonlinear constitutive relatjonships are [1]:

$$
\begin{aligned}
& \bar{F}=\alpha_{1} \bar{J}+\frac{1}{2} \alpha_{2} \bar{\nabla} \times \bar{\omega}-\beta_{1}\left(\bar{\omega}-\frac{1}{2} \bar{\nabla} \times \bar{U}\right) \times \bar{J} \\
& \bar{\lambda}^{A}=\alpha_{2} \bar{J}+\frac{1}{2} \alpha_{3} \bar{\nabla} \times \bar{\omega} ;
\end{aligned}
$$




$$
\begin{aligned}
& \bar{\lambda}^{S}=\alpha_{6}(\bar{\nabla} \cdot \bar{\omega}) \bar{I}+\frac{1}{2} \alpha_{7}\left[(\bar{\nabla} \bar{\omega})+(\bar{\nabla} \bar{\omega})^{T}-\frac{2}{3} \bar{\nabla} \cdot \bar{\omega} \bar{I}\right] \\
& \bar{\tau}^{A}=\alpha_{4}\left(\bar{\omega}-\frac{1}{2} \bar{\nabla} \times \bar{U}\right) ; \quad \bar{\tau}^{S}=\frac{1}{2} \alpha_{5}\left[\overline{\nabla U}+(\overline{\nabla U})^{T}\right]
\end{aligned}
$$

where $\alpha_{1}, \ldots, \alpha_{7}, \beta_{1}$ - closing coefficients that are determined based on experimental data.

The system of equations (1.1) with closing relationships (1.2) can be solved when $\alpha_{i}, \beta_{1}$ and boundary conditions for $\bar{U}, \bar{\omega}$ and $\varphi$ are known.

For the surface of cylinders, no-flux boundary conditions are often used:

$$
\bar{U}=0 ; \quad \bar{\omega}=k \frac{1}{2} \bar{\nabla} \times \bar{U} ; \quad \bar{n} \cdot \bar{J}=0,
$$

where the parameter $k$ characterizes the influence of the flow on the particle rotation. In the case that the constitutive coefficients $\alpha_{1}, \ldots, \alpha_{7}, \beta_{1}$ are constants, inserting (1.2) into equations (1.1), the following system of equations can be derived:

$$
\begin{aligned}
& \bar{\nabla} \cdot \bar{U}=0 ; \quad \bar{U}=\varphi \bar{U}_{1}+(1-\varphi) \bar{U}_{2} ; \\
& \rho \frac{d \varphi}{d t}=-\bar{\nabla} \cdot \bar{J} ; \quad \bar{J}=\rho \varphi\left(\bar{U}_{1}-\bar{U}\right) ; \quad \rho=\mathrm{const} \\
& \rho \frac{d \bar{U}}{d t}=\rho \bar{g}-\bar{\nabla} p-\alpha_{4} \bar{\nabla} \times \bar{\omega}+\frac{\alpha_{5}-\alpha_{4}}{2} \nabla^{2} \bar{U} \\
& j \frac{d \bar{\omega}}{d t}+j\left(\frac{\bar{J}}{A} \cdot \bar{\nabla}\right) \bar{\omega}=-\alpha_{4} \bar{\omega}+\frac{\alpha_{4}}{2} \bar{\nabla} \times \bar{U}+\alpha_{2} \bar{\nabla} \times \bar{J}+\frac{\alpha_{3}}{2}\left[\bar{\nabla}(\bar{\nabla} \cdot \bar{\omega})-\nabla^{2} \bar{\omega}\right]+ \\
& +\left(\alpha_{6}-\frac{\alpha_{7}}{3}\right) \bar{\nabla} \cdot(\bar{\nabla} \cdot \bar{\omega}) \bar{I}+\frac{\alpha_{7}}{2}\left(\nabla^{2} \bar{\omega}+\bar{\nabla}(\bar{\nabla} \cdot \bar{\omega})\right) ; \\
& \frac{d \bar{J}}{d t}+\bar{J} \cdot \bar{\nabla} \bar{U}=-\rho \varphi(1-\varphi)\left[\alpha_{1} \bar{J}+\frac{\alpha_{2}}{2} \bar{\nabla} \times \bar{\omega}-\beta_{1}\left(\bar{\omega}-\frac{1}{2} \bar{\nabla} \times \bar{U}\right) \times \bar{J}+a_{4} \bar{\nabla} \varphi\right]
\end{aligned}
$$

where $\bar{I}$ is a identity tensor of the second range and $a_{4}=\frac{1}{1-\varphi}\left(\frac{\partial \mu_{1}}{\partial \varphi}\right)_{p, T}$.

\section{MOTION EQUATION SYSTEM FOR THE CASE OF ROTATING PARTICLES}

Now consider the flow of viscous fluid with the rigid spherical rotating particles in the gap between two vertically coaxial cylinders. Using cylindrical coordinate system, the coordinate axes are denoted by $r, \theta, z$ corresponding respectively to radius, angle of rotation and vertical axis of cylinders. When inner cylinder is rotating with an angular velocity $\Omega_{0}$ and the outer cylinder is immovable, it is assumed to be considered the following case:

$* \bar{U}=\{0, u, 0\} ; \bar{\omega}=\{0,0, \omega\} ; \bar{J}=\left\{J_{r}, J_{\theta}, 0\right\} ;$

${ }^{*} \omega, \mathrm{u}, \mathrm{J}_{r}, \mathrm{~J}_{\theta}, \varphi$ are functions of $r$ and $t$. 
In this case the system of equations (1.4) has the form as follows:

$$
\begin{aligned}
& \rho \frac{\partial \varphi}{\partial t}=-\frac{1}{r} \frac{\partial}{\partial r}\left(r J_{r}\right) \\
& \rho \frac{\partial u}{\partial t}=\frac{\partial}{\partial r}\left\{a_{2}\left[\omega-\frac{1}{2 r} \frac{\partial}{\partial r}(r u)\right]\right\}+\frac{1}{r} \frac{\partial}{\partial r}\left(a_{1} r \frac{\partial u}{\partial r}\right)-\frac{u}{r}\left(\frac{\partial a_{1}}{\partial r}+\frac{a_{1}}{r}\right) \\
& j\left(\frac{\partial \omega}{\partial t}+\frac{J_{r}}{A} \frac{\partial \omega}{\partial r}\right)=a_{2}\left[\omega-\frac{1}{2 r} \frac{\partial}{\partial r}(r u)\right]+\frac{1}{r} \frac{\partial}{\partial r}\left(a_{3} r \frac{\partial \omega}{\partial r}\right)+\alpha_{2} \frac{1}{r} \frac{\partial}{\partial r}\left(r J_{\theta}\right)+J_{\theta} \frac{\partial \alpha_{2}}{\partial r} \\
& \frac{\partial J_{r}}{\partial t}-2 \frac{u J_{\theta}}{r}=-\rho \varphi(1-\varphi)\left\{\alpha_{1} J_{r}+\beta_{1} J_{\theta}\left[\omega-\frac{1}{2 r} \frac{\partial}{\partial r}(r u)\right]+\alpha_{4} \frac{\partial \varphi}{\partial r}\right\} \\
& \frac{\partial J_{\theta}}{\partial t}+J_{r} \frac{\partial u}{\partial r}+\frac{u J_{r}}{r}=-\rho \varphi(1-\varphi)\left\{\alpha_{1} J_{\theta}-\frac{\alpha_{2}}{2} \frac{\partial \omega}{\partial r}-\beta_{1} J_{r}\left[\omega-\frac{1}{2 r} \frac{\partial}{\partial r}(r u)\right]\right\}
\end{aligned}
$$

where $a_{1}=\frac{\alpha_{5}}{2} ; a_{2}=\alpha_{4} ; a_{3}=\frac{\alpha_{7}-\alpha_{3}}{2}$.

\section{NUMERICAL SOLUTION OF STEADY-STATE CASE}

A steady state of system of equations (2.2) is:

$$
\begin{aligned}
& \alpha_{4} \frac{d}{d r}\left[\omega-\frac{1}{2 r} \frac{d}{d r}(r u)\right]+\alpha_{5} \frac{d}{d r}\left[\frac{1}{2 r} \frac{d}{d r}(r u)\right]=0 \\
& \alpha_{4}\left[\omega-\frac{1}{2 r} \frac{d}{d r}(r u)\right]+\left(\frac{\alpha_{7}-\alpha_{3}}{2}+\frac{\alpha_{2}^{2}}{2 \alpha_{1}}\right) \frac{1}{r} \frac{d}{d r}\left(r \frac{d \omega}{d r}\right)=0 \\
& \frac{d \varphi}{d r}=\frac{\alpha_{2}}{2 a_{4} \alpha_{1}} \frac{d \omega}{d r}\left\{2 \frac{u}{\rho \varphi(1-\varphi) r}-\beta_{1}\left[\omega-\frac{1}{2 r} \frac{d}{d r}(r u)\right]\right\} \\
& J_{\theta}=\frac{\alpha_{2}}{2 \alpha_{1}} \frac{d \omega}{d r} ; \quad J_{r}=0
\end{aligned}
$$

with boundary conditions:

- at surface of inner cylinder $r=R_{i}$

$$
\left.u\right|_{R_{i}}=\Omega_{0} R_{i} ;\left.\quad \omega\right|_{R_{i}}=k \Omega_{x}\left(R_{i}\right) ; \quad \Omega_{x}=\frac{1}{2 r} \frac{d}{d r}(r u)
$$

- at surface of outer cylinder $r=R_{0}$

$$
\left.u\right|_{R_{0}}=0 ;\left.\quad \omega\right|_{R_{0}}=k \Omega_{x}\left(R_{0}\right)
$$

In addition there is a condition of particle number conservation:

$$
\frac{2}{R_{0}^{2}-R_{i}^{2}} \int_{R_{i}}^{R_{0}} \varphi r d r=\varphi_{0} .
$$

The system of equations (3.1)-(3.3) is closed and represented a steady state of flow of above presented problem. 


\section{Numerical result.}

The numerical results of (3.1)-(3.3) with

$\frac{\alpha_{5}}{2}=5.10^{-3} ; \alpha_{4}=5.10^{-4} ; \frac{\alpha_{7}-\alpha_{3}}{2}=1.10^{-3} ; a_{4}=15.10^{-5} ; a_{5}=\frac{\alpha_{2} \beta_{1}}{2 \alpha_{1}}=5.10^{-3}$;

$R_{i}=0.064(\mathrm{~m}) ; R_{0}=0.254(\mathrm{~m}) ; \Omega_{0}=1.5 ; \varphi_{0}=0.5 ;$

for cases $k=0,0.5,1$ are demonstrated in Fig. 1 - 4, in which length scale is normalised to $R_{0}$, velocities to $R_{i} \Omega_{0}$ and time to $\Omega_{0}^{-1}$, respectively.

The distributions of particle for the cases $k=1.0, \varphi_{0}=0.5$, when $\Omega_{0}$ has the values: $\Omega_{0}=1.5,3.0,6.0,15.0$, are showed in Fig. 4 .

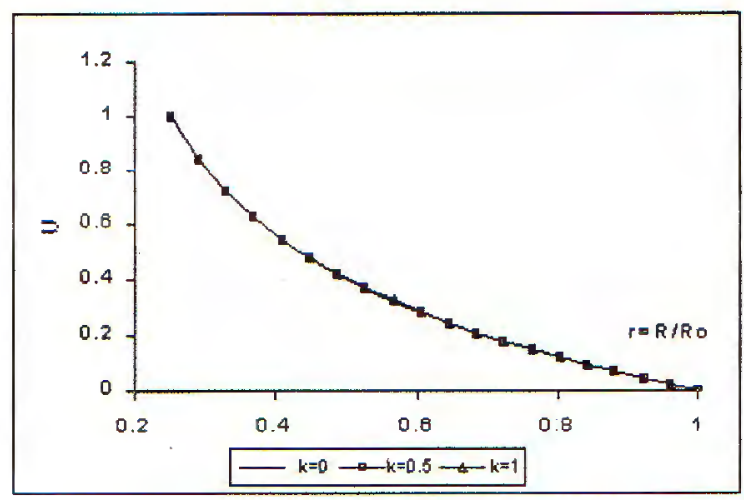

Fig. 1. Suspension velocity distribution in radial direction for the cases $k=0,0.5,1$

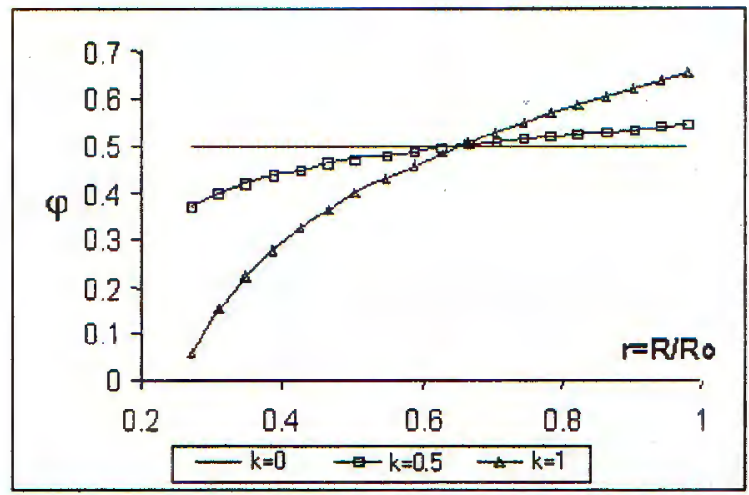

Fig. 3. Profiles of particle concentration for the cases $k=0,0.5,1$.

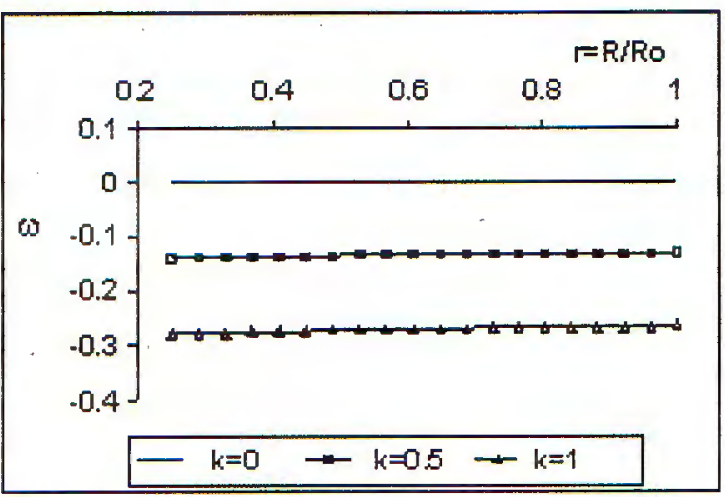

Fig. D. Rotational velocity of particles for the cases $k=0,0.5,1$

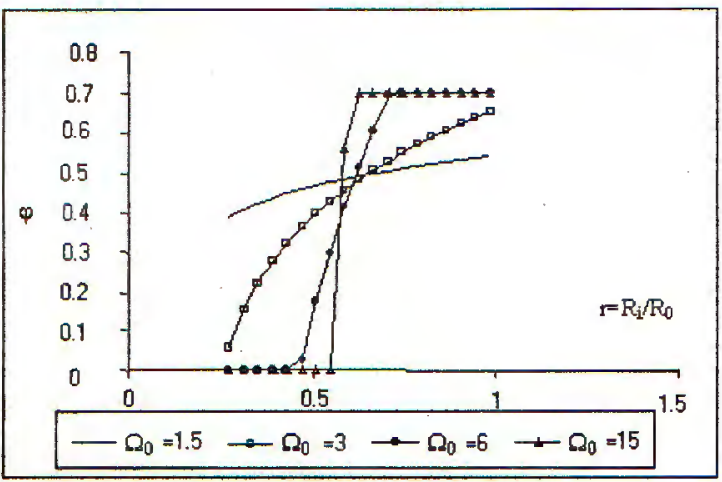

Fig. 4. Profiles of $\varphi$ for the cases $\Omega_{0}=1.5,3,6$ and 15 when $k=1 ; \varphi_{0}=0.5$

\section{COMPARISON WITH EXPERIMENTAL DATA}

The experimental apparatus that was described in Philip R. J., Armstrong R. C., 1992 [2] consisted of a rotating cylinder, concentric in a fixed outer tube . The inner, outer radii and the high of the cylinder were $0.64 \mathrm{~cm}, 2.38 \mathrm{~cm}$ and $25 \mathrm{~cm}$, respectively. 
The rotational velocity of the inner cylinder varied between $0.28-1.95$ round per second (rps). The particles were made by Polymethyl Methacrylate (PMMA) with mean diameter $a=0.675 \mathrm{~mm}$ and density of $\rho=1.182 \mathrm{~g} / \mathrm{cm}^{3}$. The suspending liquid was a Newtonian oil with the same density of the particles and has viscosity $\eta=4.95 \mathrm{~Pa} .5$ at $23.15^{\circ} \mathrm{C}$.

The measured data of particles concentrations in a steady state with stational rotational velocity of inner cylinder for three cases of initial particle concentrations $\varphi_{0}=0.45$; 0.50 and 0.55 are denoted by the curves $\varphi-0.45, \varphi-0.50, \varphi-0.55$ respectively on Figs. 5 -8 .

Since the rotational velocity of inner cylinder was not mentioned in [2] so as a test of the model, we do the numerical experiment (i.e. solve numerically) the equation system (3.1)-(3.3) with the parameters similar to the experiment and in four rotational velocities $\Omega=1.0 ; 1.5 ; 2.0$ and $2.5 \mathrm{rps}$. The results are demonstrated by the curves $T 1-0.45, T 2-$ $0.50, T 3-0.55$ respectively to the cases $\varphi_{0}=0.45 ; 0.50$ and 0.55 in Fig. $5-8$.

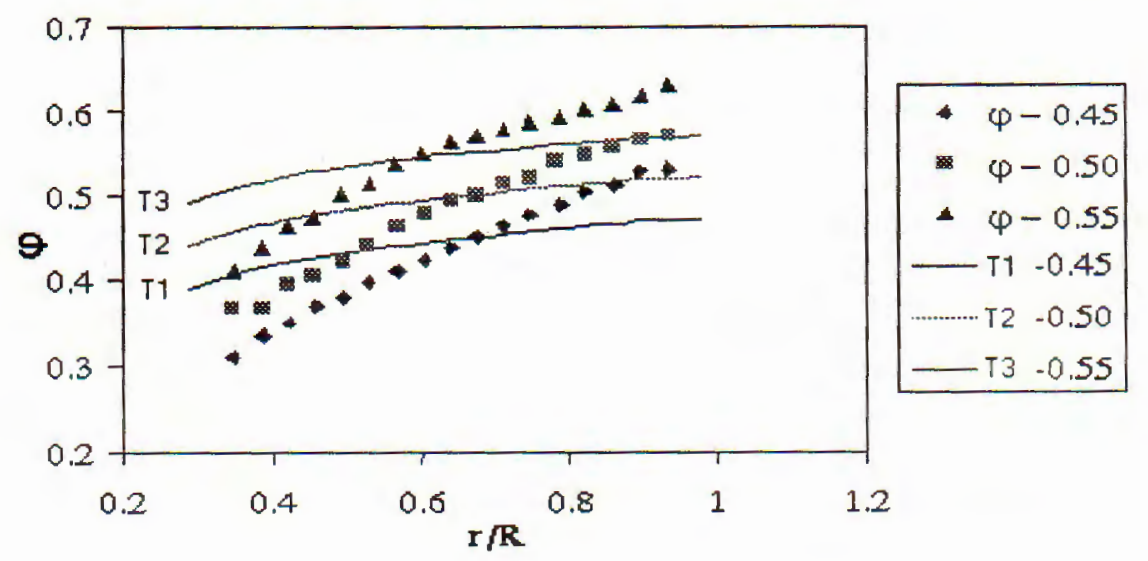

Fig. 5. A comparison of the numerical results with the experimental data [2] for $\Omega=1.0 \mathrm{rps}$ and different $\varphi$

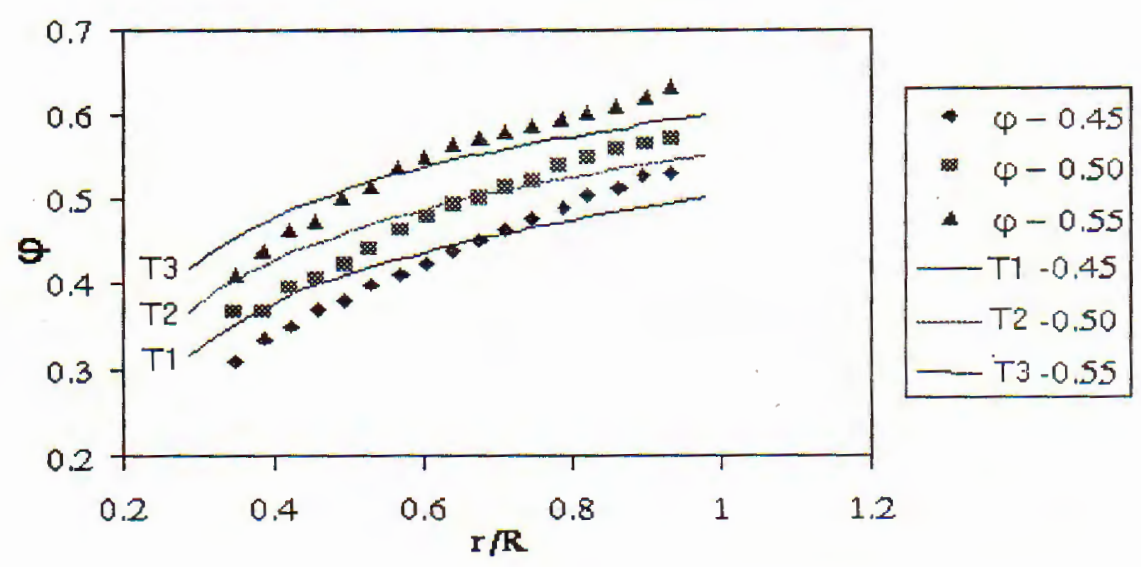

Fig.6. A comparison of the numerical results with the experimental data for $\varphi_{0}=0.45 ; 0.5$ and 0.55 when $\Omega=1.5 \mathrm{rps}$ 


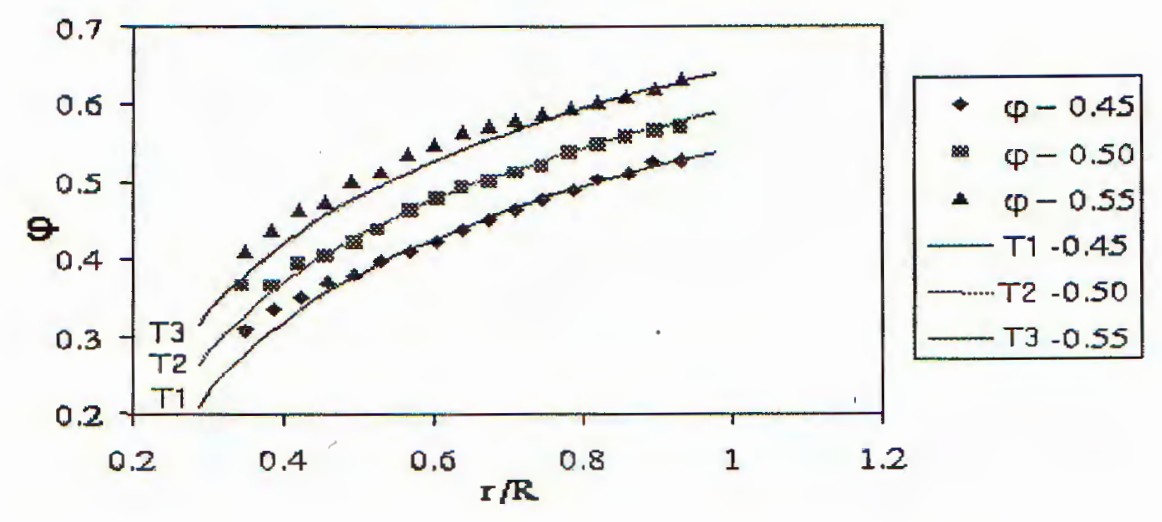

Fig. 7. A comparison of the numerical results with the experimental data for $\varphi_{0}=0.45 ; 0.5$ and 0.55 when $\Omega=2.0 \mathrm{rps}$



Fig. 8. A comparison of the numerical results with the experimental data for $\varphi_{0}=0.45 ; 0.5$ and 0.55 when $\Omega=2.5$ rps

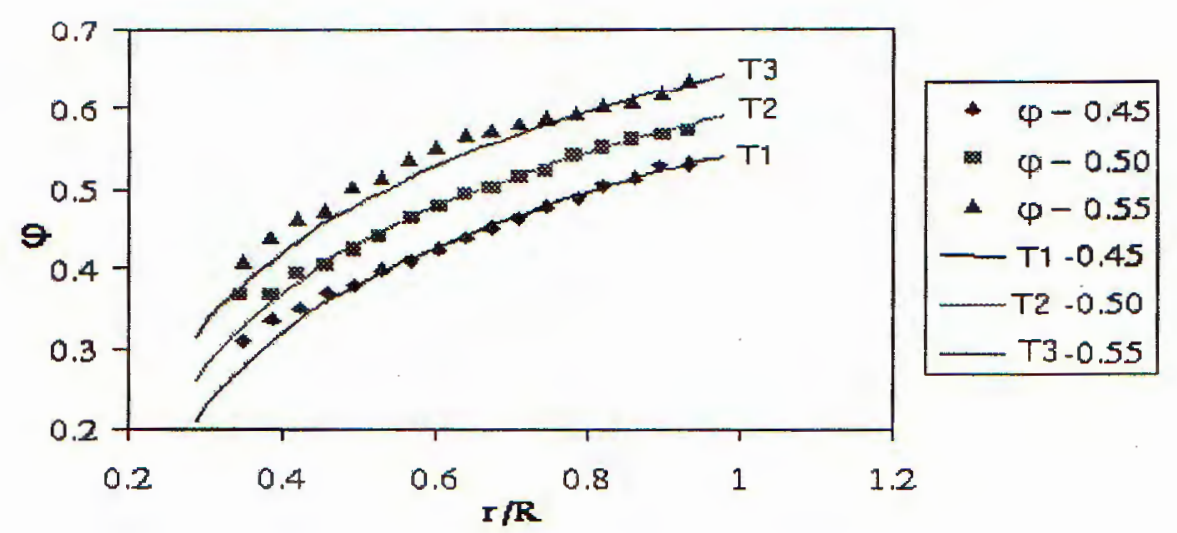

Fig. 9. A comparison of the numerical results with the experimental data for $\varphi_{0}=0.45 ; 0.5$ and 0.55 when $\Omega=1.95 \mathrm{rps}$ 


\section{DISCUSSION AND CONCLUSIONS}

- The influence of $k$ on particle concentration clearly presented in Fig. 3. When $k=0$, i.e. rotation of fluid was not transfered to particles, that leads to $\varphi=\varphi_{0}=$ const. When $k=0.5,1.0$ particle distribution inscreases from inner to the outer cylinder.

- In Fig. 4, the change of the rotational velocity of the inner cylinder effects on the local volume fraction of particles. For $\Omega_{0} \geq 12$ all particles concentrate nearby the outer cylinder and makes the particle volume fraction to be maximum and equals $\sim 0.7$. This value is close to the suggested value by Phillip et al. in [2].

- It is clear in Fig. 8 that the good agreement between the numerical result of the model and measured concentration profile of particle is achieved at $\Omega=1.95 \mathrm{rps}$.

The demonstrated investigation is one of the first stage for understanding and applying of the generalized diffusion theory for description of two phase flow of viscouse fluid with rotationable particles. The numerical tests show that determination of value and range of the used coefficients in the model are very important and more investigation is required.

Acknowledgements. This work was financially supported by the Vietnamese National Foundation for Basic Research in Natural Sciences. The author woud like to thank Prof. Dr. of sciences Nguyen Van Diep for his help and corrections.

\section{REFERENCES}

1. Nguyễn Hồng Phan, Nguyễn Văn Điệp, Lý thuyết khuếch tán suy rộng cho chuyển động của các hạt cầu cứng quay được trong chất lỏng nhớt giữa hai ống hình trụ, Tuyển tập công trình Hội nghi Co học Thưy khí Toàn quốc năm 2003.

2. Ronal J. Phillips, Robert C. Armstrong and Robert A. Brown, A constituitive equation for concentrated suspensions that accounts for shear-induced particle migration, Phys. Fluids A, 1992, 4.

Received August 1, 2005

Revised October 8, 2005

\section{ÁP DỰG LÝ THUYẾT KHUỂCH TÁN SUY RộNG TÍNH TOÁN PHÂN BỐ CƯA CÁC HẠT TRONG DÒNG CHẢY GIỮA HAI ỐNG TRỤ ĐỒNG TÂM THĂNG ĐỨNG}

Báo cáo trình bày sự áp dụng lý thuyết khuếch tán suy rộng mô tả chuyển động của các hạt cầu rẳn quay được trong dòng chất lơng nhớt sử dụng các hệ thức đóng kín dạng phi tuyến. Đã thiết lập bài toán chuyển động của hỗn hợp giữa hai ống trụ đồng tâm thẳng đứng, ống trụ trong quay, ống trụ ngoài đứng yên và giải số cho trường hợp dòng chảy dừng. Kết quả tính toán được so sánh với số liệu thực nghiệm của Phillip (1992). 